Pacific Journal of Mathematics

A MULTIMOVE INFINITE GAME WITH LINEAR PAYOF 


\section{A MULTIMOVE INFINITE GAME WITH LINEAR PAYOFF}

\section{Leonard D. Berkovitz ANd Melvin Dresher}

1. Introduction. Games can be classified in terms of the number of moves by each player-unimove or multimove-and in terms of the number of choices-finite or infinite-available at each move. The original work of von Neumann [2] on the existence and structure of solutions of games was, in effect, restricted to unimove finite games. Later, Ville [3] proved the existence of optimal strategies for unimove infinite games with continuous payoff function.

Except for games with 'perfect information, multimove finite games have been analyzed only very recently; and multimove infinite games with an arbitrary number of moves have hardly been touched upon.

In this paper, we analyze a multimove infinite game with a linear payoff function. The game is symmetric in every respect except that the initial conditions of the two players are different. We prove that one player has an optimal pure strategy and that the other player must randomize on the strategies. The optimal strategies and game value are derived.

Although this game had its origin in a military problem concerning allocation of resources among several tasks, it is presented here solely for its mathematical interest. A complete discussion of the military problem and its solution is given in [1].

2. Description of game. We shall analyze the following multimove zero-sum two-person game. At the $n$th move, or stage of the game, Blue has resources given by the state variable $p_{n}$ and assigns a value to each of two tactical variables under his control, $x_{n}$ and $u_{n}$, subject to the constraints

$$
x_{n} \geq 0, \quad u_{n} \geq 0, \quad x_{n}+u_{n} \leq p_{n} .
$$

At the same time, Red has resources given by the state variable $q_{n}$ and controls the values of the tactical variables $y_{n}$ and $w_{n}$, subject to the constraints

$$
y_{n} \geq 0, \quad w_{n} \geq 0, \quad y_{n}+w_{n} \leq q_{n} .
$$

Let us number the moves from the end of the game; i.e., the $n$th move means $n$ moves to the end of the game. The state variables at the $(n-1)$-st move are defined by

Received February 10, 1959, and in revised form September 14, 1959. 


$$
\begin{aligned}
& p_{n-1}=\max \left[0, p_{n}-\max \left(0, y_{n}-u_{n}\right)\right], \\
& q_{n-1}=\max \left[0, q_{n}-\max \left(0, x_{n}-w_{n}\right)\right] .
\end{aligned}
$$

The payoff to Blue is given by

$$
\sum_{n=1}^{N}\left[\left(p_{n}-x_{n}-u_{n}\right)-\left(q_{n}-y_{n}-w_{n}\right)\right],
$$

where $N$ is the number of moves in the game.

The play of the game proceeds by first making the $N$ th move, then the $(N-1)$-st move, $\cdots$, the second move, and the first move. An $n$th move of the game consists of a choice by Blue of $x_{n}$ and $u_{n}$ satisfying (2.1) and simultaneously a choice by Red of $y_{n}$ and $w_{n}$ satisfying (2.2). We assume that each player knows the manner in which the game proceeds from stage to stage; namely, each player has the information expressed by equations (2.3). We also assume that at each stage of the game both players know the state variables and the entire past history of the play; that is, at the $n$th move, both players know $N, p_{N}, q_{N}$, and also know $x_{i}, u_{i}, y_{i}, w_{i}$ for $i=N, N-1, \cdots, n+2, n+1$. It follows that $p_{i}, q_{i}$, for $i=N, N-1, \cdots, n+1, n$, are known at the $n$th move.

The strategies of the game in normal form will be defined inductively on the number of moves. First, a strategy for Blue in a one-move game is a point $X_{1}=\left(x_{1}, u_{1}\right)$, where $x_{1} \geq 0, u_{1} \geq 0$, and $x_{1}+u_{1} \leq p_{1}$. Similarly a strategy for Red in a one move game is a point $Y_{1}=\left(y_{1}, w_{1}\right)$ where $y_{1} \geq 0, w_{1} \geq 0$, and $y_{1}+w_{1} \leq q_{1}$. Now let $\sigma_{N}$ be a strategy for Blue in an $N$-move game. Of course, $\sigma_{N}$ is a function of $p_{N}$ and $q_{N}$. Then, in a game of $N+1$ moves, at the $(N+1)$-st move Blue chooses a point $X_{N}=\left(x_{N+1}, u_{N+1}\right)$ in the triangle $\Delta_{N+1}$ defined by

$$
x_{N+1} \geq 0, \quad u_{N+1} \geq 0, \quad x_{N+1}+u_{N+1} \leq p_{N+1},
$$

and simultaneously Red chooses a point $Y_{N+1}=\left(y_{N+1}, w_{N+1}\right)$ in the triangle $D_{N+1}$ defined by

$$
y_{N+1} \geq 0, \quad w_{N+1} \geq 0, \quad y_{N+1}+w_{N+1} \leq q_{N+1} .
$$

These choices yield the state variables $p_{N}$ and $q_{N}$, by equations (2.3). A strategy $\sigma_{N+1}$ for Blue in the $(N+1)$ move game is then defined as a choice $X_{N+1}$ in $\Delta_{N+1}$ and a function $\Phi_{N}$ that associates, with each point $\left(x_{N+1}, u_{N+1}, y_{N+1}, w_{N+1}\right) \equiv\left(X_{N+1}, Y_{N+1}\right)$ in the product space $\Delta_{N+1} D_{N+1}$ a strategy $\sigma_{N}$ in the $N$-move game. Thus $\sigma_{N+1}$ can be written as

$$
\sigma_{N+1}=\left(X_{N+1} ; \Phi_{N}\right)=\left(x_{N+1}, u_{N+1} ; \Phi_{N}\right),
$$

where $\Phi_{N}$ assigns the strategy $\sigma_{N}$ to the point $\left(x_{N+1}, u_{N+1}, y_{N+1}, w_{N+1}\right)$.

In a like manner, a strategy $\tau_{N+1}$ for Red in the $(N+1)$-move game is defined as a choice $Y_{N+1}$ and a function $\bar{\psi}_{N}$ that associates, with each 
$\left(X_{N+1}, Y_{N+1}\right)$, a strategy $\tau_{N}$ in the $N$-move game. Thus we have

$$
\tau_{N+1}=\left(Y_{N+1} ; \bar{\psi}_{N}\right)=\left(y_{N+1}, w_{N+1} ; \psi_{N}\right) \text {. }
$$

3. Solution of game. The main result of this paper is the following:

Theorem 1. If $N=1$ or 2 , the value of the game is given by

$$
V_{N}\left(p_{N}, q_{N}\right)=N\left(p_{N}-q_{N}\right) \text {. }
$$

Blue has an optimal pure strategy:

$$
\bar{x}_{m}=\bar{u}_{m}=0 \text { for } m \leq N \text {. }
$$

Red has an optimal pure strategy:

$$
\bar{y}_{m}=\bar{w}_{m}=0 \text { for } m \leq N \text {. }
$$

If $N \geq 3$, the value of the game is given by the $(N-2)$-piecewiselinear function.

$$
V_{N}\left(p_{N}, q_{N}\right)=a_{N}^{i} p_{N}-b_{N}^{i} q_{N}, \quad i=1,2, \cdots, N-2,
$$

where the constants $a_{N}^{i}$ and $b_{N}^{i}$ are positive and monotone decreasing in $i$ for fixed $N$; the value of the superscript $i$ is determined by the ratio $p_{N} / q_{N}$. The optimal strategies for the two players are as follows:

(i) At move $m=1,2$ (counting from the end) the players choose

$$
\bar{x}_{m}=\bar{u}_{m}=\bar{y}_{m}=\bar{w}_{m}=0 \text {. }
$$

(ii) At move $m=3$, if, $p_{3} \geq q_{3}$, then Blue chooses $\bar{x}_{3}, \bar{u}_{3}$ such that

$$
\begin{aligned}
& q_{3} \leq \bar{x}_{3} \min \left(\frac{p_{3}+q_{3}}{2}, \frac{3 q_{3}}{2}\right), \\
& \bar{u}_{3}=\bar{x}_{3}-q_{3} .
\end{aligned}
$$

Red chooses either $\bar{y}_{3}=q_{3}$ or $\bar{w}_{3}=q_{3}$, each with probability $1 / 2$.

(iii) At the $(m+1)$-st move, where $3 \leq m \leq N-1$, if $p_{m+1} \geq q_{m+1}$, then the ratio $p_{m+1} / q_{m+1}$ determines an integer $i, 1 \leq i \leq m-1$, and Blue chooses

$$
\begin{aligned}
& \bar{x}_{m+1}=\frac{\left(2 m-a_{m}^{i}\right) p_{m+1}-\left(m-2 b_{m}^{i}\right) q_{m+1}}{m+b_{m}^{i}} \\
& \bar{u}_{m+1}=p_{m+1}-\bar{x}_{m+1}, \quad \text { for } i=1,2, \cdots, m-2,
\end{aligned}
$$

and

$$
\bar{x}_{m+1}=\left(2-\frac{1}{b_{m}^{m-2}}\right) q_{m+1},
$$




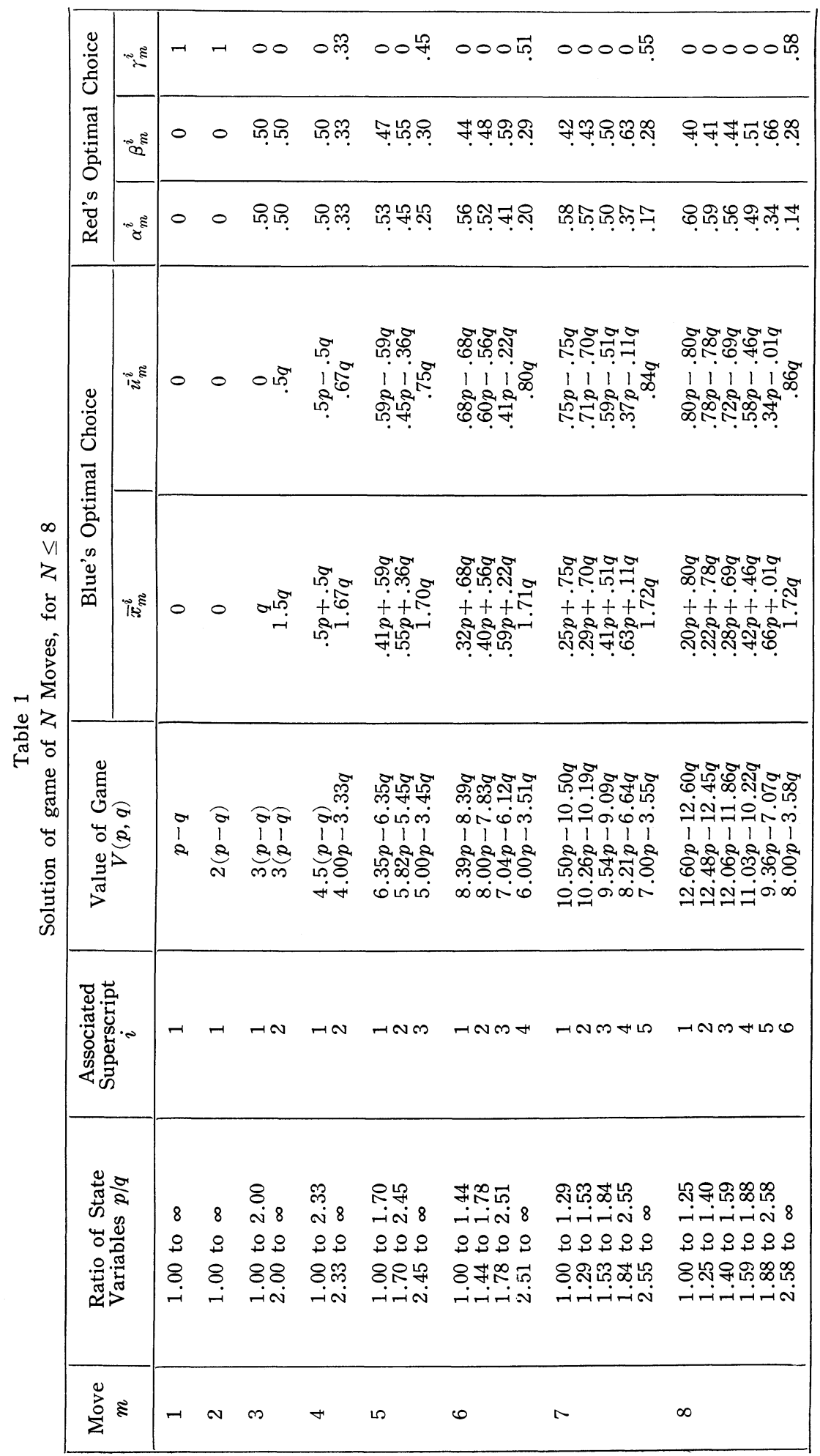




$$
\bar{u}_{m+1}=\left(1-\frac{1}{m}\right) q_{m+1}, \quad \text { for } i=m-1,
$$

where the constants $a_{m}^{i}$ and $b_{m}^{i}$ are those associated with a game of length $m$ and initial condition $p_{m}, q_{m}$. Red chooses either $y_{m+1}$ or $w_{m+1}=q_{m+1}$ with probabilities $\alpha_{m}^{i}=b_{m}^{i} /\left(m+b_{m}^{i}\right)$ and $\beta_{m}^{i}=m /\left(m+b_{m}^{i}\right)$, respectively, for $i=1,2, \cdots, m-2$; however, if $i=m-1$, Red chooses $y_{m+1}=q_{m+1}$ with probability $\alpha_{m}^{i}=1 / m$, or $w_{m+1}=q_{m+1}$ with probability $\beta_{m}^{i}=1 / b_{m}^{m-2}$, or $y_{m+1}=w_{m+1}=0$ with probability $\gamma_{m}^{i}=1-1 / m-1 /\left(b_{m}^{m-2}\right)$.

The proof of Theorem 1 will be carried out by induction on $N$, the number of moves of the game. In the course of this argument, recursive definitions will be given for the constants $a_{N}^{i}$ and $b_{N}^{i}$. As an illustration of the theorem, Table 1 shows the solutions for games with eight or less moves.

4. A three-part sufficiency condition with mixed strategies. From the statement of the theorem, it is seen that mixed strategies will have to be introduced, at least for Red. However, it is sufficient to introduce a restricted class of mixed strategies in order to prove the theorem.

For a game of one move, a mixed strategy for Red is a probability distribution $G_{1}$ over $D_{1}$. Now suppose $G_{N}$ is a mixed strategy for Red in a game of $N$ moves and state variables $p_{N}$ and $q_{N}$. Then a probability distribution $g_{N+1}$ over $D_{N+1}$ and a function $\psi_{N+1}$ that associates $\left(x_{N+1}, u_{N+1}, y_{N+1}, w_{N+1}\right)$ with $G_{N}$ is a mixed strategy $G_{N+1}$ in the $(N+1)$ move game. Thus we may write the mixed strategy as

$$
G_{N+1}=\left(g_{N+1}, \psi_{N}\right) \text {. }
$$

Mixed strategies $F_{N+1}$ for Blue are defined similarly by a distribution function $f_{N+1}$ and a function $\phi_{N}$, and can be written as

$$
F_{N+1}=\left(f_{N+1}, \phi_{N}\right) \text {. }
$$

Let $\hat{F}_{N+1}$ denote a mixed strategy for Blue in the $(N+1)$-move game in which he selects $X_{N+1}=\left(x_{N+1}, u_{N+1}\right)$ with probability 1 at the $(N+1)$-st move. Let $\hat{G}_{N+1}$ denote a mixed strategy for Red in which he selects $Y_{N+1}=\left(y_{N+1}, w_{N+1}\right)$ with probability 1 at the $(N+1)$-st move.

Suppose that Theorem 1 is valid for games of length $N=n$. Let $F_{n}^{*}$ and $G_{n}^{*}$ be optimal strategies for Blue and Red, respectively. Let $\phi_{n}^{*}, \psi_{n}^{*}$ denote the functions that associate $\left(x_{n+1}, u_{n+1}, y_{n+1}, w_{n+1}\right)$ with $F_{n}^{*}, G_{n}^{*}$, respectively. Suppose, further, that $p_{n+1} \geq q_{n+1}$ (from symmetry, it suffices to consider this case only).

The theorem asserts that at the $(n+1)$-st move Blue's optimal choice is a point $\left(x_{n+1}, u_{n+1}\right)$ that is determined by the ratio $p_{n+1} / q_{n+1}$. Denote this point by 


$$
X_{n+1}^{*}=\left(x_{n+1}^{*}, u_{n+1}^{*}\right)=\left(x_{n+1}^{*}\left(p_{n+1}, q_{n+1}\right), u_{n+1}^{*}\left(p_{n+1}, q_{n+1}\right)\right),
$$

and let $F_{n+1}^{*}=\left(X_{n+1}^{*}, \phi_{n}^{*}\right)$.

The theorem further asserts that Red chooses $\left(y_{n+1}, w_{n+1}\right)$ to be

$$
Y_{n+1}^{(1)} \equiv(q, 0), Y_{n+1}^{(2)} \equiv(0, q), Y_{n+1}^{(3)} \equiv(0,0),
$$

with probabilities $\alpha_{n+1}, \beta_{n+1}$, and $\gamma_{n+1}=\left(1-\alpha_{n+1}-\beta_{n+1}\right)$, respectively, the values of $\alpha_{n+1}$ and $\beta_{n+1}$ being determined by the ratio $p_{n+1} / q_{n+1}$. Denote this distribution in $\Delta_{n+1}$ by $g_{n+1}^{*}\left(p_{n+1}, q_{n+1}\right)$, and set $G_{n+1}^{*}=$ $\left(g_{n+1}^{*}\left(p_{n+1}, q_{n+1}\right), \psi_{n}^{*}\right)$.

Define

$$
L_{n+1}\left(X_{n+1}, Y_{n+1}\right) \equiv p_{n+1}-x_{n+1}-u_{n+1}-q_{n+1}+y_{n+1}+w_{n+1}
$$

and

$$
M_{n+1}\left(X_{n+1}, Y_{n+1}\right) \equiv L_{n+1}\left(X_{n+1}, Y_{n+1}\right)+V_{n}\left(p_{n}, q_{n}\right),
$$

where $p_{n}, q_{n}$ are obtained from $p_{n+1}, q_{n+1}$ by means of (2.3) and the choices $x_{n+1}, u_{n+1}, y_{n+1}, w_{n+1}$. Let $E_{N}\left(F_{N}, G_{N}\right)$ denote the expected payoff of the game of length $N$ if Blue chooses a strategy $F_{N}$ and Red chooses a strategy $G_{N}$. Then

$$
\begin{aligned}
E_{n+1}\left(\hat{F}_{n+1}^{*}, \hat{G}_{n+1}\right) & =L_{n+1}\left(X_{n+1}^{*}, Y_{n+1}\right)+E_{n}\left(F_{n}^{*}, G_{n}\right) \\
& \geq M_{n+1}\left(X_{n+1}^{*}, Y_{n+1}\right), \text { for all } Y_{n+1},
\end{aligned}
$$

where $\psi_{n}\left(X_{n+1}^{*}, Y_{n+1}\right)=G_{n}$. Furthermore, we have

$$
\begin{aligned}
E_{n+1}\left(\hat{F}_{n+1}, G_{n+1}^{*}\right)= & \alpha_{n+1}\left[L_{n+1}\left(X_{n+1}, Y_{n+1}^{(1)}\right)+E_{n}\left(F_{n}, G_{n}^{*}\right)\right] \\
& +\beta_{n+1}\left[L_{n+1}\left(X_{n+1}, Y_{n+1}^{(2)}\right)+E_{n}\left(F_{n}, G_{n}^{*}\right)\right] \\
& +\left(1-\alpha_{n+1}-\beta_{n+1}\right)\left[L_{n+1}\left(X_{n+1}, Y_{n+1}^{(3)}\right)+E_{n}\left(F_{n}, G_{n}^{*}\right)\right] \\
\leq & \alpha_{n+1} M_{n+1}\left(X_{n+1}, Y_{n+1}^{(1)}\right)+\beta_{n+1} M_{n+1}\left(X_{n+1}, Y_{n+1}^{(2)}\right) \\
& +\left(1-\alpha_{n+1}-\beta_{n+1}\right) M_{n+1}\left(X_{n+1}, Y_{n+1}^{(3)}\right),
\end{aligned}
$$

for all $X_{n+1}$, where $\phi_{n}\left(X_{n+1}, Y_{n+1}\right)=F_{n}$.

The validity of the following lemma is now apparent.

Lemma 1. Given that Theorem 1 is true for $N=n$, to prove the theorem for $N=n+1$ with initial conditions $p_{n+1} \geq q_{n+1}$, it suffices to exhibit the $X_{n+1}^{*}, \alpha_{n+1}$, and $\beta_{n+1}$ for which

$$
\begin{gathered}
E_{n+1}\left(\hat{F}_{n+1}^{*}, G_{n+1}^{*}\right)=V_{n+1}\left(p_{n+1}, q_{n+1}\right), \\
M_{n+1}\left(X_{n+1}^{*}, Y_{n+1}\right) \geq V_{n+1}\left(p_{n+1}, q_{n+1}\right)
\end{gathered}
$$

for all $Y_{n+1}$, and

$$
\begin{aligned}
& \alpha_{n+1} M_{n+1}\left(X_{n+1}, Y_{n+1}^{(1)}\right)+\beta_{n+1} M_{n+1}\left(X_{n+1}, Y_{n+1}^{(2)}\right) \\
& \quad+\left(1-\alpha_{n+1}-\beta_{n+1}\right) M_{n+1}\left(X_{n+1}, Y_{n+1}^{(3)}\right) \leq V_{n+1}\left(p_{n+1}, q_{n+1}\right)
\end{aligned}
$$


for all $X_{n+1}$.

5. Some special cases. It will also be useful to tabulate the information given by equations (2.3). We may assume that $p_{n} \geq q_{n}$, whence $y_{n}-u_{n}>p_{n}$ is impossible and the equations (2.3) can be tabulated as follows, where the subscript $n$ is suppressed:

Table 2

Determination of Values of State Variables $p_{n-1}$ and $q_{n-1}$

\begin{tabular}{c|c|c|c}
\hline Region in $(X, Y)$ Space & $\begin{array}{c}\text { Region } \\
\text { Number }\end{array}$ & $p-1$ & $q-1$ \\
\hline$y-u<0, \quad x-w<0$ & I & $p$ & $q$ \\
$y-u<0,0 \leq x-w \leq q$ & II & $p$ & $q-x+w$ \\
$y-u<0, \quad x-w>q$ & III & $p$ & 0 \\
$0 \leq y-u \leq p, \quad x-w \leq 0$ & IV & $p-y+u$ & $q$ \\
$0 \leq y-u \leq p, 0 \leq x-w \leq q$ & V & $p-y+u$ & $q-x+w$ \\
$0 \leq y-u \leq p, \quad x-w>q$ & VI & $p-y+u$ & 0 \\
\hline
\end{tabular}

Games of length $N=1,2,3$ will now be discussed. From the statement of the theorem, it is clear that separate arguments are needed for $N=1,2$, and for $N \geq 3$.

For $N=1$, an examination of the payoff (2.4) shows that optimal play for Blue is to choose $x_{1}=u_{1}=0$, and that optimal play for Red is to choose $y_{1}=w_{1}=0$.

As a consequence of Lemma 1 , for $N=2$ it suffices to consider

$$
M_{2}\left(X_{2}, Y_{2}\right)=p_{2}-x_{2}-u_{2}-q_{2}+y_{2}+w_{2}+\left(p_{1}-q_{1}\right),
$$

with $p_{2} \geq q_{2}$. Using Table 2 and dropping the subscript 2, we may write this

$$
M(X, Y)= \begin{cases}2(p-q)-(x+u)+(y+w) & \text { in region } \mathrm{I}, \\ 2(p-q)-u+w & \text { in region } \mathrm{II}, \\ 2 p-q-(x+u)+(y+w) & \text { in region III, } \\ 2(p-q)-x+w & \text { in region } \mathrm{IV}, \\ 2(p-q) & \text { in region } \mathrm{V}, \\ 2 p-q-x+w, & \text { in region } \mathrm{VI},\end{cases}
$$

where the region in the $(X, Y)$ space for which each expression on the right is valid is that given in Table 2. It now follows that the optimal choices at the second move are

$$
(\bar{x}, \bar{u})=(0,0) \text { for Blue; }(\bar{y}, \bar{w})=(0,0) \text { for Red; }
$$

and that $V_{2}=2\left(p_{2}-q_{2}\right)$. Thus the theorem is proved for $N=2$.

For $N=3$, it suffices to consider

$$
M_{3}\left(X_{3}, Y_{3}\right)=p_{3}-x_{3}-u_{3}-q_{3}+y_{3}+w_{3}+2\left(p_{2}-q_{2}\right),
$$


where $p_{3} \geq q_{3}$. It follows from Table 2 that, dropping the subscript 3, we may write $M_{3}\left(X_{3}, Y_{3}\right)$ as

$$
A p-B q+\varepsilon_{1} x+\varepsilon_{2} u+\varepsilon_{3} y+\varepsilon_{4} V,
$$

where the values of $A, B, \varepsilon_{i}= \pm 1, i=1, \cdots, 4$ depend on the region of the $(X, Y)$ space. To prove the theorem for $N=3$, we use (5.1) and Lemma 1 to verify that the choices given for the third move are actually optimal.

Games of length $N=3$, and $N=4$, as can be seen from Table 1 , are somewhat transitional in character, and do not exhibit all the characteristics of games of arbitrary length. For $N=5$, however, all of the characteristics of the game's structure become apparent. For the reader who wishes to gain further insight into the structure of the game, as well as to obtain a motivation for the general induction step, the computation of the functions $m_{i}(x, u) \equiv \min _{Y} M_{i}\left(X_{i}, Y_{i}\right), i=3,4,5$, is recommended. By way of illustration we give $m_{5}(x, u)$, valid for $1 \leq p / q \leq 7 / 3$. The authors found the construction of a diagram quite instructive, and also indicative of the form of $m_{5}(x, u)$ for $p / q \geq 7 / 3$. We have that $m_{5}(x, u)$ is given as follows, (where the subscript 5 is omitted):

(i) $5 p-q-x-u$, if $x \geq 17 q / 10, u \geq 3 q / 4$;

(ii) $5 p-20 q / 3+7 x / 3-u$, if $2 q-3 p / 7 \leq x \leq 17 q / 10,5 x-6 u \leq 4 q$;

(iii) $11 p / 2-9 q+7 x / 2-u$, if $q \leq x \leq 2 q-3 p / 7$,

(iv) $11(p-q) / 2-u$, if $0 \leq x \leq q, 9 u+7 x \geq 7 q$,

$$
9 x-8 u \leq 10 q-p
$$

(v) $11 p / 2-9 q+7(x+u) / 2$, if $x \geq 0, u \geq 0$,

$$
14 x+24 u \geq 3 p+11 q
$$

$9 u+7 x \leq 7 q, 7 x+3 u \leq 10 q-3 p ;$

(vi) $5 p+22 q / 3+7 x / 3+3 u$, if $x \leq q, u \geq 0$,

$$
7 x+3 u \geq 10 q-3 p, 14 x+24 u \leq 3 p+11 q
$$

(vii) $5 p-4 q-x+3 u$ if $x \geq q, u \geq 0,5 x-6 u \geq 4 q$,

$$
9 x-8 u \geq 10 q-p \text {. }
$$

6. Definitions and properties of constants. The first step of the proof of Theorem 1 is to define the sequences $\left\{a_{N}^{i}\right\},\left\{b_{N}^{i}\right\},\left\{\lambda_{N}^{i}\right\}$. To this end, consider the following sequences defined in the manner and order indicated:

$$
\begin{gathered}
a_{3}^{1}=3, a_{n+1}^{n-1}=a_{n}^{n-2}+1 ; n \geq 3 ; \\
b_{3}^{1}=3, b_{n+1}^{n-1}=4-\frac{1}{a_{n}^{n-2}}-\frac{1}{b_{n}^{n-2}}, n \geq 3 ; \\
b_{n}^{0}=a_{n}^{0}=0, n \geq 3 ;
\end{gathered}
$$




$$
\begin{gathered}
\left.\begin{array}{c}
a_{n+1}^{i}=\frac{a_{n}^{n-2}\left(2 b_{n}^{i}+a_{n}^{i}\right)}{b_{n}^{i}+a_{n}^{n-2}} \\
b_{n+1}^{i}=\frac{3 a_{n}^{n-2} b_{n}^{i}}{b_{n}^{i}+a_{n}^{n-2}}
\end{array}\right\} \quad i \geq 1 ; n=i+2, i+3, \cdots ; \\
\lambda_{n}^{n-1}=+\infty, n=3,4,5, \cdots ; \\
\lambda_{3}^{1}=1, \\
\lambda_{n+1}^{n-1}=3-\frac{1}{a_{n}^{n-2}}-\frac{1}{b_{n}^{n-2}}=b_{n+1}^{n-1}-1, n=3,4,5, \cdots ; \\
\lambda_{n}^{i}=\frac{b_{n}^{i-1}-b_{n}^{i}}{a_{n}^{i-1}-a_{n}^{i}}, n \geq 4 ; \quad i=1,2,3, \cdots, n-3 .
\end{gathered}
$$

The following properties of the foregoing sequences will be useful in the proof of the theorem; indications of the derivations of the properties are given after the listing:

$$
a_{n}^{n-2}=n,
$$

$$
n \geq 3 \text {; }
$$

$$
a_{n}^{1}=b_{n}^{1} \text {, }
$$$$
n \geq 3 \text {; }
$$

$$
4>b_{n+1}^{n-1}>b_{n}^{n-2} \geq 3,
$$$$
n \geqq 3 ;
$$

$$
a_{n}^{i} \geq b_{n}^{i}>0 \text {, }
$$$$
i=1,2, \cdots, n-2 \text {; }
$$$$
n=3,4,5, \cdots \text {; }
$$

$$
n \leq a_{n}^{i}<2 n,
$$$$
i=1, \cdots, n-2 \text {; }
$$$$
n=3,4,5, \cdots, \text {; }
$$

$$
\left.\begin{array}{l}
a_{n}^{i}<a_{n}^{i-1}, \\
b_{n}^{i}<b_{n}^{i-1},
\end{array}\right\}
$$$$
n \geq 3 ; i=2,3, \cdots, n-2 \text {; }
$$

$$
\lambda_{n+1}^{i}=\frac{3 n \lambda_{n}^{i}}{\left(2 n-a_{n}^{i}\right) \lambda_{n}^{i}+\left(n+b_{n}^{i}\right)},
$$$$
n \geq 3 ; i=1,2, \cdots, n-2 \text {; }
$$

$$
3>\lambda_{n+1}^{n-1}>\lambda_{n}^{n-2} \geq 2,
$$

$$
n \geq 3 \text {; }
$$

$$
\lambda_{n}^{i+1}>\lambda_{n}^{i} \text {, }
$$$$
n \geq 3 ; i=1,2, \cdots, n-2 \text {; }
$$

$$
\lambda_{n+1}^{i} \leq \lambda_{n}^{i},
$$$$
n \geq 3 ; i=1, \cdots, n-2 \text {. }
$$

Statements (6.9)-(6.11) follow from the definitions and from trivial inductive arguments.

Inequalities (6.12) and (6.13) are proved by induction on $n, n \geq i+2$, for each fixed $i$.

The monotonicity properties in (6.14) are established as follows. The monotonicity of $\left\{b_{n+1}^{i}\right\}, i=1, \cdots, n-2$, follows, by induction on $n$, from the monotonicity of $\left\{b_{n}^{i}\right\}, i=1, \cdots, n-2$. To show that $b_{n+1}^{n-2}>b_{n+1}^{n-1}$, it suffices to show that $b_{n+1}^{n-2} \geq 4$. This inequality, however, is obvious 
from (6.5) and (6.11). When we compute $a_{n+1}^{i-1}-a_{n+1}^{i}, i=2,3, \cdots, n-2$, we obtain

$$
\frac{n}{\left(n+b_{1}^{i-1}\right)\left(n+b^{i}\right)}\left[b^{i-1}\left(2 n-a^{i}\right)-b^{i}\left(2 n-a^{i-1}\right)+n\left(a^{i-1}-a^{i}\right)\right],
$$

where the subscript $n$ is omitted. From the inductive hypothesis that $\left\{a_{n}^{i}\right\}$ is monotone decreasing in $i$, and from (6.13), the bracketed expression in turn is seen to be larger than the positive quantity

$$
b^{i-1}\left(2 n-a^{i-1}\right)-b^{i}\left(2 n-a^{i-1}\right)=\left(b^{i-1}-b^{i}\right)\left(2 n-a^{i-1}\right) .
$$

Furthermore, for $n \geq 3$, by (6.1), (6.4), and (6.11) we have

$$
a_{n+1}^{n-2}-a_{n+1}^{n-1}=\frac{b_{n}^{n-2}(n-1)-1}{b^{n-2}+n} \geq \frac{3(n-1)-n}{b^{n-2}+n}>0 .
$$

Thus the fact that $\left\{a_{n+1}^{i}\right\}$ is monotone decreasing in $i$ is established.

To prove (6.15), we use (6.4) and (6.5) in the definition (6.8) of $\lambda_{n+1}^{i}$.

Inequality (6.16) is obvious, and (6.17) is established inductively as follows. Suppose $\lambda_{n}^{i+1}>\lambda_{n}^{i}$ for $i=1, \cdots, n-1$. Consider

$$
\frac{\lambda_{n+1}^{i+1}}{\lambda_{n+1}^{i}}=\frac{\lambda^{i+1}\left[\left(2 n-a^{i}\right) \lambda^{i}+\left(n+b^{i}\right)\right]}{\lambda^{i}\left[\left(2 n-a^{i+1}\right) \lambda^{i+1}+\left(n+b^{i+1}\right)\right]}, \quad i=1,2, \cdots, n-3,
$$

where the subscript $n$ is omitted. To prove that this ratio exceeds 1 , it therefore suffices to show that

$$
n\left(\lambda^{i+1}-\lambda^{i}\right)+\lambda^{i+1} b^{i}-\lambda^{i} b^{i+1}>\lambda^{i} \lambda^{i+1}\left(a^{i}-a^{i+1}\right) .
$$

Upon replacing $\lambda^{i+1}$ on the right by its definition (6.8), we see that this last inequality is equivalent to $\left(n+b^{i}\right)\left(\lambda^{i+1}-\lambda^{i}\right)>0$, the validity of which follows from the inductive hypothesis. The chain

$$
\lambda_{n+1}^{n-2}=\frac{3 n \lambda^{n-2}}{\left(\lambda^{n-2}+1\right) n+b^{n-2}}=\frac{3 n \lambda^{n-2}}{b^{n-2}(n+1)} \leq \frac{3 \lambda^{n-2}}{b^{n-2}}<\lambda^{n-2}<\lambda_{n+1}^{n-1}
$$

completes the proof of (6.17).

It is seen from (6.15) that to verify (6.18) it suffices to show that

$$
\lambda_{n}^{i} \geq \frac{2 n-b_{n}^{i}}{2 n-a_{n}^{i}}, n \geq 3 ; \quad i=1,2, \cdots, n-2 .
$$

This inequality is shown to hold by induction on $i$, as follows. For $i=1$, equality is obvious. Suppose that the inequality holds for $i=k$. It is then seen to hold for $i=k+1,1 \leq i \leq n-3$, by writing (omitting the subscript $n$ )

$$
\begin{aligned}
\lambda^{k+1}\left(2 n-a^{k+1}\right) & -\left(2 n-b^{k+1}\right)=\lambda^{k+1}\left(2 n-a^{k}\right)-\left(2 n-b^{k}\right) \\
& +\lambda^{k+1}\left(a^{k}-a^{k+1}\right)-\left(b^{k}-b^{k+1}\right) \\
& >\lambda^{k}\left(2 n-a^{k}\right)-\left(2 n-b^{k}\right) \geq 0 .
\end{aligned}
$$


7. Miscellaneous preparations. For $N \leq 3$, Theorem 1 has been proved in $\S 5$. The theorem will now be proved inductively for arbitrary $N>3$. Suppose then that it has been established for $N=n \geq 3$. It is required to show that it holds for $N=n+1$.

In order to simplify notation, for the remainder of the proof we shall omit the subscript $n$. Thus the symbol $a_{n}^{i}$, say, will be written merely as $a^{i}$, the symbol $\lambda_{n+1}^{i-1}$ as $\lambda_{1}^{i-1}, b_{n-1}^{i+1}$ as $b_{-1}^{i+1}$, etc.

From the symmetry of Theorem 1, it is clear that it suffices to consider the case $p_{1} \geq q_{1}$. Define $X_{1}^{*}=X_{1}^{*}\left(p_{1} / q_{1}\right)$ as follows:

$$
X_{1}^{*}=\bar{X}_{1}^{i} \equiv\left(\bar{x}_{1}^{i}, \bar{u}_{1}^{i}\right) \text { if } \lambda_{1}^{i} \leq \frac{p_{1}}{q_{1}} \leq \lambda_{1}^{i+1}, \quad i=1, \cdots, n-1,
$$

where

$$
\begin{array}{ll}
\bar{x}_{1}^{i}=\frac{\left(2 n-a^{i}\right) p_{1}-\left(n-2 b^{i}\right) q_{1}}{b^{i}+n}, \bar{u}_{1}^{i}=p_{1}-\bar{x}_{1}^{i}, \quad i=1, \cdots, n-2, \\
\bar{x}_{1}^{n-1}=\left(2-\frac{1}{b^{n-2}}\right) q_{1}, & \bar{u}_{1}^{n-1}=\left(1-\frac{1}{n}\right) q_{1} .
\end{array}
$$

Define $\alpha_{1}=\alpha_{1}\left(p_{1} / q_{1}\right)$ and $\beta_{1}=\beta_{1}\left(p_{1} / q_{1}\right)$ thus:

(a) if $\lambda_{1}^{i} \leq \frac{p_{1}}{q_{1}} \leq \lambda_{1}^{i+1}$, $1 \leq i \leq n-2$ then

$$
\alpha_{1}=\alpha_{1}^{i}=\frac{b^{i}}{n+b^{i}}, \quad \beta_{1}=\beta_{1}^{i}=\frac{n}{n+b^{i}} ;
$$

(b) if $\frac{p_{1}}{q_{1}} \geq \lambda_{1}^{n-1}$,

then

$$
\alpha_{1}^{n-1}=\frac{1}{n}, \quad \beta_{1}^{n-1}=\frac{1}{b^{n-2}} .
$$

Clearly, $\alpha_{1}^{i}>0, \beta_{1}^{i}>0$ for all $i$ satisfying $i \leq 1 \leq n-1 ; \alpha^{i}+\beta^{i}=1$ for all $i$ satisfying $1 \leq i \leq n-2$; and $\alpha^{i}+\beta^{i}<1$ for $i=n-1$. Thus $\alpha_{1}$ and $\beta_{1}$ are probabilities. Lemma 2 will show that $X_{1}^{*}$ is an admissible choice for Blue and will furnish some useful bounds for $\bar{x}_{1}^{i}$ and $\bar{u}_{1}^{i}$.

Lemma 2. The point $X_{1}^{*}$ is an admissible choice of strategic variable for Blue. Furthermore, for all $i$ satisfying $1 \leq i \leq n-1$, we have

$$
\begin{aligned}
& q_{1} \leq \bar{x}_{1}^{i} \leq 2 q_{1}, \\
& 0 \leq \bar{u}_{1}^{i}<q_{1} .
\end{aligned}
$$


Since $X_{1}^{*}$ is defined piecewise, the first step in showing that $X_{1}^{*}$ is admissible is to show that the pieces come together-i.e., that $X_{1}^{*}$ is well defined. Substitution of $p_{1}=\lambda_{1}^{n-1} q_{1}$ into the definition (7.1) of $\bar{x}_{1}^{n-2}$, and application of (6.7), show that if $p_{1}=\lambda_{1}^{n-1} q_{1}$, then $\bar{x}_{1}^{n-2}=\bar{x}_{1}^{n-1}$. Similarly, it is seen that $\bar{u}_{1}^{n-2}=\bar{u}_{1}^{n-1}$ for $p_{1}=\lambda_{1}^{n-1} q_{1}$. Substitution of $p_{1}=\lambda_{1}^{i} q_{1}, i=1, \cdots, n-2$, into the definition (7.1) of $\bar{x}_{1}^{i}$, and use of (6.15), show that, for $p_{1}=\lambda_{1}^{i} q_{1}$,

$$
\bar{x}_{1}^{i}=\left(2-\frac{\lambda_{1}^{i}}{\lambda^{i}}\right) q_{1} .
$$

Substitution of $p_{1}=\lambda_{1}^{i+1} q_{1}$, and use of (6.15), show that, for $p_{1}=\lambda_{1}^{2+1} q_{1}$ s $i=1, \cdots, n-3$,

$$
\bar{x}_{1}^{i}=\left(2-\frac{\lambda_{1}^{i+1}}{\lambda^{i+1}}\right) q_{1}
$$

Thus $X_{1}^{*}$ is well defined, and for $\lambda_{1}^{i} \leq p_{1} / q_{1} \leq \lambda_{1}^{i+1}, i=1,2, \cdots, n-3$,

$$
\left(2-\frac{\lambda_{1}^{i}}{\lambda^{i}}\right) q_{1} \leq \bar{x}_{1}^{i} \leq\left(2-\frac{\lambda_{1}^{i+1}}{\lambda^{i+1}}\right) q_{1},
$$

with equality on the left occurring for $p_{1}=\lambda_{1}^{i} q_{1}$, and on the right for $p_{1}=\lambda_{1}^{i+1} q_{1}$. Similarly, we obtain

$$
\left(2-\frac{\lambda_{1}^{n-2}}{\lambda^{n-2}}\right) q_{1} \leq \bar{x}_{\mathrm{I}}^{n-2} \leq \bar{x}_{1}^{n-1} .
$$

Clearly, (7.3) implies $\bar{x}_{1}^{i} \geq 0, \bar{u}_{1}^{i} \geq 0$ for all $i$ satisfying $1 \leq i \leq n-1$. By definition, $\bar{x}_{1}^{i}+\bar{u}_{1}^{i}=p_{1}$ for $1 \leq i \leq n-2$; and for $i=n-1$, we obtain $\bar{x}_{1}^{n-1}+\bar{u}_{1}^{n-1}=\lambda_{1}^{n-1} q_{1} \leq p_{1}$. Thus the proof of the lemma depends on the proof of (7.3). For $i=n-1,(7.3)$ is obvious. The inequality (6.18) implies $\lambda_{1}^{i} \leq \lambda^{i}$, whence it follows that $\left(2-\lambda_{1}^{i} / \lambda^{i}\right) q_{1} \geq q_{1}$ for $i=1$, $2, \cdots, n-2$. Clearly, the inequality $\left(2-\lambda_{1}^{i} / \lambda^{i}\right) q_{1}<2 q_{1}$ holds for $i=$ $1, \cdots, n-2$. Hence, we obtain $q_{1} \leq \bar{x}_{1}^{i}<2 q_{1}$ for $i=1, \cdots, n-2$.

To verify $0 \leq \bar{u}_{1}^{i}<q_{1}$ for $1 \leq i \leq n-2$, we substitute from the definition of $\bar{x}_{1}^{i}$ into the definition $\bar{u}_{1}^{i}=p_{1}-\bar{x}_{1}^{i}$ and obtain

$$
\bar{u}_{1}^{i}=\frac{\left(b^{i}+a^{i}-n\right) p_{1}+\left(n-2 b^{i}\right) q_{1}}{\left(b^{i}+n\right)}, \quad 1 \leq i \leq n-2 .
$$

Hence, showing that $\bar{u}_{1}^{i}<q_{1}$ is equivalent to showing that $p_{1} / q_{1}<3 b^{i} /\left(b^{i}+\right.$ $\left.a^{i}-n\right)$. Since $p_{1} / q_{1} \leq \lambda_{1}^{i+1}$, it clearly suffices to show that $\lambda_{1}^{i+1}<3 b^{i} /\left(b^{i}+\right.$ $a^{i}-n$ ). If $i=n-2$ this inequality is obvious. If $i \leqq n-3$, then by using (6.15) and (6.8) this inequality becomes

$$
b^{i}-\lambda^{i+1}\left(a^{i}-n\right)>0, \quad i=1,2, \cdots, n-3 .
$$

Since $a^{n-2}=n$, it follows from (6.8) that for $i=n-3$ the expression on 
the left of this inequality is positive. Further, the left hand member is a monotone decreasing function of $i$, as can be seen by forming the difference of the left-hand side for superscripts $i-1$ and $i$ and getting $\left(\lambda^{i+1}-\lambda^{i}\right)\left(a^{i}-n\right)>0$. This inequality follows from (6.13) and (6.17). Thus it follows that $\bar{u}_{1}^{i}<q_{1}$. The inequality $\bar{u}_{1}^{i} \geq 0$, follows from

$$
\left(b^{i}+a^{i}-n\right) p_{1}+\left(n-2 b^{i}\right) q_{1} \geq\left(a^{i}-b^{i}\right) q_{1} \geq 0 ; \quad 1 \leq i \leq n-2,
$$

and the lemma is proved.

It follows from Lemma 1 and the definitions of $X_{1}^{*}, \alpha_{1}, \beta_{1}$, and from the inductive hypothesis to the effect that Theorem 1 is valid for $N=n$, that the validity of Theorem 1 will be established if (4.1), (4.2), and (4.3) of Lemma 1 are shown to hold for this $X_{1}^{*}, \alpha_{1}, \beta_{1}$. The next sections of the proof will be devoted to the verification of these three statements.

In the course of this verification, it will be necessary to compute

$$
\begin{aligned}
M_{1}\left(X_{1}, Y_{1}\right) & =L_{1}\left(X_{1}, Y_{1}\right)+V(p, q) \\
& =L_{1}\left(X_{1}, Y_{1}\right)+a^{j} p-b^{j} q, \quad j=1, \cdots, n-2,
\end{aligned}
$$

explicitly in terms of $p_{1}, q_{1}, X_{1}, Y_{1}$, for certain choices of $X_{1}, Y_{1}$. For any given fixed initial condition $\left(p_{1}, q_{1}\right)$, an integer $1 \leq i \leq n-1$ is determined by the inequality $\lambda_{1}^{i} \leq p_{1} / q_{1} \leq \lambda_{1}^{i+1}$. Each choice $\left(X_{1}, Y_{1}\right)$ by the players falls into one of the six regions enumerated in Table $2^{1}$ and determines $p$ and $q$ and hence an integer $1 \leq j \leq n-2$ via the inequality $\lambda^{j} \leq p / q \leq \lambda^{j+1}$. It is this integer $j$ that appears in (7.6). Clearly, $j$ is a function of $p_{1}, q_{1}, X_{1}$, and $Y_{1}$. In computing $M_{1}\left(X_{1}, Y_{1}\right)$ explicitly in terms of the initial conditions and choices $X_{1}, Y_{1}$, it will thus be necessary to take into account the region of the $\left(X_{1}, Y_{1}\right)$ space and the superscript $j$. The statement " $\left(X_{1}, Y_{1}\right)$ leads to case III ${ }^{j_{0}}$ "' will mean that, for the initial condition being considered, the pair $\left(X_{1}, Y_{1}\right)$ falls into region III of the $\left(X_{1}, Y_{1}\right)$ space and the ratio $p / q$ is such that $j=j_{0}$. At first glance, it appears that there are $6(n-2)$ cases. Actually, not all of these cases are possible; and since some specialization of $X_{1}, Y_{1}$ will occur, not all of the possible cases will be encountered.

8. Verification, first part of sufficiency condition. We divide the discussion into two cases.

Case 1. $p_{1} / q_{1} \geq \lambda_{1}^{n-1}$.

For this initial condition, it is readily seen from Table 2 that $\left(X_{1}^{*}, Y_{1}^{(1)}\right)$ leads to case $\mathrm{VI}^{n-2},\left(X_{1}^{*}, Y_{1}^{(2)}\right)$ leads to case $\mathrm{II}^{n-2}$, and $\left(X^{*}, Y_{1}^{(3)}\right)$ leads to case $\mathrm{III}^{n-2}$. It then follows by straightforward computation

1 The table is given for passage from $n$ to $n-1$, whereas the present situation is for passage from $n+1$ to $n$. The adjustment of subscripts is left to the reader. 
and the definition of $\S 6$ that

$$
M_{1}\left(X_{1}^{*}, Y_{1}^{(1)}\right)=M_{1}\left(X_{1}^{*}, Y_{1}^{(2)}\right)=M_{1}\left(X_{1}^{*}, Y_{1}^{(3)}\right)=V_{1}\left(p_{1}, q_{1}\right) .
$$

The equality $E_{1}\left(\hat{F}^{*}, G_{1}^{*}\right)=V_{1}\left(p_{1}, q_{1}\right)$ now follows from

$$
\begin{aligned}
& E_{1}\left(\hat{F}_{1}^{*}, G_{1}^{*}\right)=\alpha_{1}\left[L_{1}\left(X_{1}^{*}, Y_{1}^{(1)}\right)+E\left(F^{*}, G^{*}\right)\right] \\
& \quad+\beta_{1}\left[L_{1}\left(X_{1}^{*}, Y_{1}^{(2)}\right)+E\left(F^{*}, G^{*}\right)\right] \\
& \quad+\left(1-\alpha_{1}-\beta_{1}\right)\left[L_{1}\left(X_{1}^{*}, Y_{1}^{(3)}\right)+E\left(F^{*}, G^{*}\right)\right] \\
& \quad=\alpha_{1} M_{1}\left(X_{1}^{*}, Y_{1}^{(1)}\right)+\beta_{1} M_{1}\left(X_{1}^{*}, Y_{1}^{(2)}\right) \\
& \quad+\left(1-\alpha_{1}-\beta_{1}\right) M_{1}\left(X_{1}^{*}, Y_{1}^{(3)} .\right.
\end{aligned}
$$

Case 2.

$$
\lambda_{1}^{i} \leq p_{1} / q_{1} \leq \lambda_{1}^{i+1},
$$$$
i=1,2, \cdots, n-2 \text {. }
$$

For this initial condition, it is immediately seen from Table 2 that $\left(X_{1}^{*}, Y_{1}^{(1)}\right)$ leads to case $\mathrm{VI}^{n-2}$, while $\left(X_{1}^{*}, Y_{1}^{(2)}\right)$ leads to case $\mathrm{II}^{j}$ for appropriate $j$. To determine the value of $j$, we first observe that $p / q=$ $p_{1} /\left(2 q_{1}-\bar{x}_{1}^{i}\right)$. It follows from (7.4) and (7.5) that $j=i$. Straightforward computation and use of the definitions in $\$ 6$ now show that

$$
M_{1}\left(X_{1}^{*}, Y_{1}^{(1)}\right)=M_{1}\left(X_{1}^{*}, Y_{1}^{(2)}\right)=V_{1}\left(p_{1}, q_{1}\right),
$$

and hence (4.1) follows as before.

9. Verification, second part of sufficiency condition. Again, as in $\S 8$, we divide the discussion into two cases.

Case 1. $p_{1} / q_{1} \geq \lambda_{1}^{n-1}$.

Since $\bar{x}_{1}^{n-2} \geq q_{1},\left(X_{1}^{*}, Y_{1}\right)$ can never fall into region I or IV of the $\left(X_{1}, Y_{1}\right)$ space. Since $\left(X_{1}^{*}, Y_{1}\right)$ lying in region $\mathrm{V}$ implies that

$$
y_{1}+w_{1} \geq \bar{x}_{1}^{n-2}+\bar{u}_{1}^{n-2}-q_{1}=\left(2-\frac{1}{b^{n-2}}+\frac{1}{n}\right) q_{1}>q_{1},
$$

this event is also impossible.

For $Y_{1}$ such that $\left(X_{1}^{*}, Y_{1}\right)$ falls in region II, we have

$$
\frac{p}{q}=\frac{p_{1}}{q_{1}-\bar{x}_{1}^{n-2}+w_{1}} \geq \frac{p_{1}}{2 q_{1}-\bar{x}_{1}^{n-2}}=b^{n-2} \frac{p_{1}}{q_{1}} \geq \lambda^{n-2},
$$

the last inequality following from (6.11) and (6.16). Hence, the superscript asociated with region II is $n-2$, and

$$
\begin{aligned}
M_{1}\left(X_{1}^{*}, Y_{1}\right)= & (n+1) p_{1}-\left(b^{n-2}-1\right) \bar{x}_{1}^{n-2}-\bar{u}_{1}^{n-2}-\left(1+b^{n-2}\right) q_{1}+y_{1} \\
& -\left(b^{n-2}-1\right) w_{1} \geq M_{1}\left(X_{1}^{*}, Y_{1}^{(2)}\right)=V_{1}\left(p_{1}, q_{1}\right),
\end{aligned}
$$

the last inequality following from (8.1).

The only superscript that can be associated with region III or VI 
is $n-2$. Having noted this, we easily see that for $\left(X_{1}^{*}, Y_{1}\right)$ in region III, $\min _{Y_{1}} M_{1}\left(X_{1}^{*}, Y_{1}\right)$ occurs for $Y_{1}=Y_{1}^{(3)}$, and so (4.2) follows from (8.1). Similarly, for $Y_{1}$ such that $\left(X_{1}^{*}, Y_{1}\right)$ is in VI,

$$
\min _{Y_{1}} M_{1}\left(X_{1}, Y_{1}\right)=M_{1}\left(X_{1}^{*}, Y_{1}^{(1)}\right)=V_{1}\left(p_{1}, q_{1}\right) \text {. }
$$

Case 2.

$$
\lambda_{1}^{i} \leq p_{1} / q_{1} \leq \lambda_{1}^{i+1}, \quad i=1, \cdots, n-2 .
$$

Since $\bar{x}_{1}^{i} \geq q_{1},\left(X_{1}^{*}, Y_{1}\right)$ cannot fall in region I or IV. If $\left(X_{1}^{*}, Y_{1}\right)$ is in region II, then we have

$$
\begin{gathered}
M_{1}\left(X_{1}^{*}, Y_{1}\right)=\left(a^{j}+1\right) p_{1}-\left(b^{j}+1\right) q_{1}+\left(b^{j}-1\right) \bar{x}_{1}^{i}-\bar{u}_{1}^{i} \\
+y_{1}-\left(b^{j}-1\right) w_{1},
\end{gathered}
$$

where $j$ is determined by the ratio $p / q=p_{1} /\left(q_{1}-\bar{x}_{1}^{i}+w_{1}\right)$. Clearly, $j$ is a nonincreasing function of $w_{1}$ alone, $j=j\left(w_{1}\right)$. From (7.4), it follows that $j\left(q_{1}\right)=i$. For each $j$, the minimum of $M_{1}\left(X_{1}^{*}, Y_{1}\right)$ is achieved at a point $Y_{1}=\left(y_{1}, w_{1}\right)$, where $y_{1}=0$ and where $w_{1}$ is the largest value of $w$ such that $\left(X_{1}^{*}, Y_{1}\right), Y_{1}=(0, w)$, leads to case $\mathrm{II}^{j}$. Hence, by the continuity of $M_{1}\left(X_{1}^{*}, Y_{1}\right)$, it follows that the minimum of $M_{1}\left(X_{1}^{*}, Y_{1}\right)$, over all $Y_{1}$ such that $\left(X_{1}^{*}, Y_{1}\right)$ is in region II, occurs at $Y_{1}^{(2)}$. Thus, using (8.2), we get

$$
M_{1}\left(X_{1}^{*}, Y_{1}\right) \geq M_{1}\left(X_{1}^{*}, Y_{1}^{(2)}\right)=V_{1}\left(p_{1}, q_{1}\right) .
$$

The only superscript possible for $\left(X_{1}, Y_{1}\right)$ in region III is $n-2$; thus for $Y_{1}$ such that $\left(X_{1}^{*}, Y_{1}\right)$ is in III, we have

$$
M_{1}\left(X_{1}^{*}, Y_{1}\right)=n p_{1}-q_{1}+\left(y_{1}+w_{1}\right) .
$$

The minimum of this expression over region III is assumed at $Y_{1}=(0,0)$ and is $n p_{1}-q_{1}$. Since we are considering the case $p_{1} / q_{1} \leq \lambda_{1}^{i+1}$, the inequality

$$
\left(b_{1}^{i}-1\right)-\lambda_{1}^{i+1}\left(a_{1}^{i}-n\right) \geq 0
$$

implies the inequality $n p_{1}-q_{1} \geq a_{1}^{i} p_{1}-b_{1}^{i} q_{1}$, and so it suffices to establish (9.1) in order to verify (4.2). With the aid of (6.8) and (6.13), it is easy to see that the left-hand side of (9.1) is a decreasing function of the superscript. Furthermore, for $i=n-2$ it follows from (6.4), (6.5), and (6.7) that the left-hand side of (9.1) is zero, and so (9.1) is verified. In the event that $Y_{1}$ is such that $\left(X_{1}^{*}, Y_{1}\right)$ is in region $\mathrm{V}$, we have

$$
\begin{aligned}
M_{1}\left(X_{1}^{*}, Y_{1}\right)= & \left.\left(a^{\jmath}+1\right) p_{1}-\left(b^{j}+1\right) q_{1}+\left(b^{j}-1\right) \bar{x}_{1}^{i}-w_{1}\right) \\
& +\left(a^{j}-1\right)\left(\bar{u}_{1}^{i}-y_{1}\right)
\end{aligned}
$$

where $j$ is determined by 


$$
\lambda^{\jmath} \leq \frac{p_{1}-y_{1}+\bar{u}_{1}^{i}}{q_{1}-\bar{x}_{1}^{i}+w_{1}} \leq \lambda^{j+1}, \quad j=1,2, \cdots, n-2 .
$$

Since $X_{1}^{*}$ is fixed, all questions concerned with determining which points in the $\left(X_{1}^{*}, Y_{1}\right)$ plane lead to the different cases $V^{j}$ are thus seen to devolve upon questions concerning point sets in the $Y_{1}$ plane. Clearly, the lines $L^{j}$,

$$
y_{1}=-\lambda^{\jmath} w_{1}+\lambda^{\jmath}\left(\bar{x}_{1}^{i}-q_{1}\right)+p_{1}+\bar{u}_{1}^{i},
$$

in the $\left(y_{1}, w_{1}\right)$ plane form a finite pencil through the point $y_{1}=p_{1}+\bar{u}_{1}^{i}$, $w_{1}=\bar{x}_{1}^{i}-q_{1}$. From the monotonicity properties of the sequence $\left\{\lambda^{i}\right\}$, it follows that for any fixed $y_{1}=c$ with $c \leq p_{1}+\bar{u}_{1}^{i}$ (and so particularly for $y_{1} \leq q_{1}$ ), as one moves along $y_{1}=c$ in the direction of increasing $w_{1}$, the lines $L^{j}$ are encountered in order of decreasing $j$, with the line $L^{n-2}$ being intercepted at a value of $w_{1}>\bar{x}_{1}^{i}-q_{1}$. Thus, the sets in the

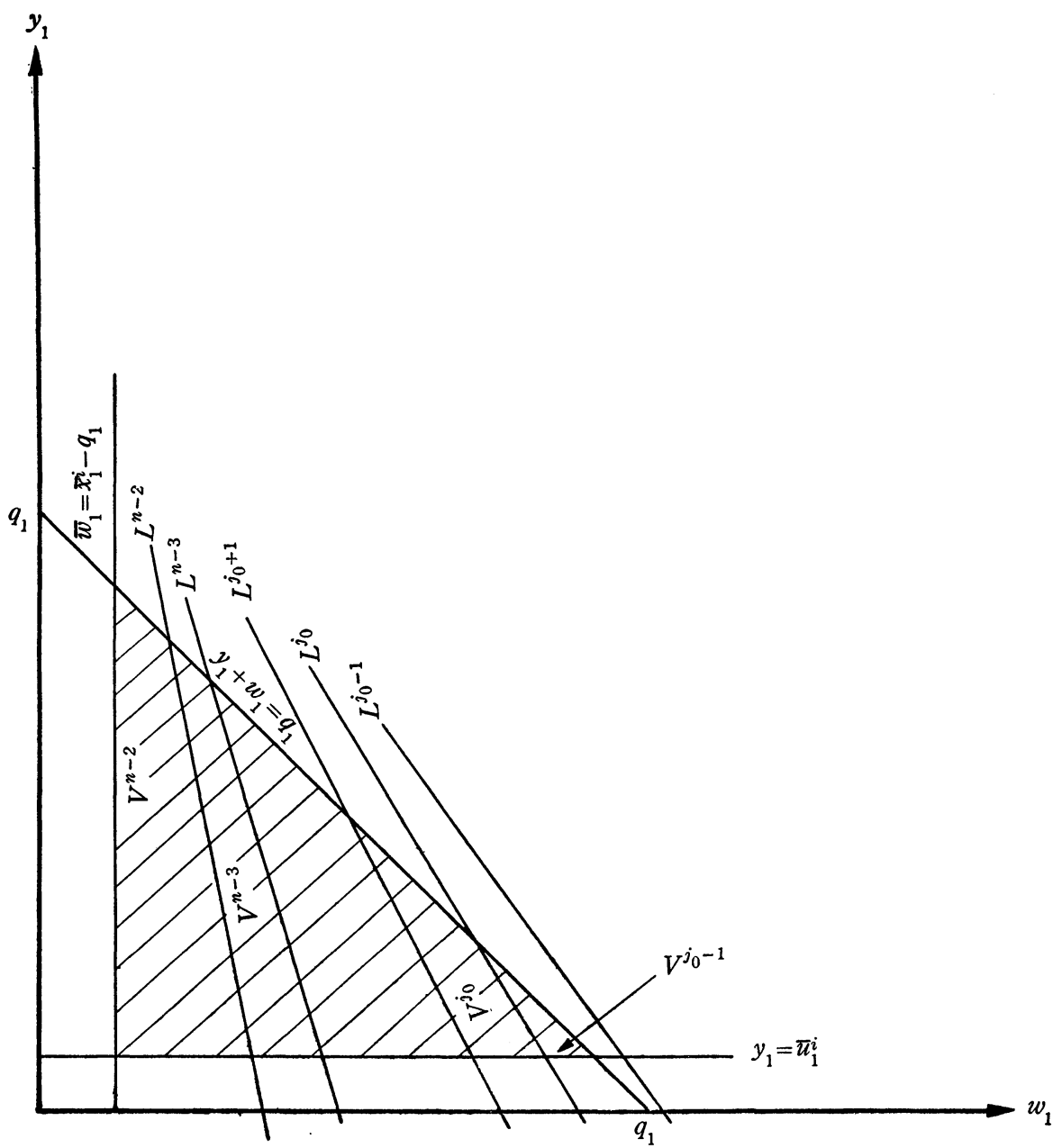

Fig. 1. 
$Y_{1}$ plane giving rise to the various cases $V^{j}$ are, in general, as indicated by the hatched regions in Fig. 5 .

From the fact that $a^{j} \geq b^{j}$, it follows that the minimum of $M_{1}\left(X_{1}^{*}, Y_{1}\right)$, over each set $V^{j}$ of Fig. 1, is assumed at the upper left-hand vertex of $V^{j}$. Hence, by the continuity of $M_{1}\left(X_{1}^{*}, Y_{1}\right)$ in $Y_{1}$, it follows that the minimum of $M_{1}\left(X_{1}^{*}, Y_{1}\right)$, over all $Y_{1}$ such that $\left(X_{1}^{*}, Y_{1}\right)$ is in $V$, is achieved at $w_{1}=\bar{x}_{1}^{i}-q_{1}, y_{1}=2 q_{1}-\bar{x}_{1}^{i}$. Substituting these values into (9.2) and using $\bar{x}_{1}^{i}+\bar{u}_{1}^{i}=p_{1}$, we see that the value of the minimum is $2 n\left(p_{1}-q_{1}\right)$. It remains to show that $2 n\left(p_{1}-q_{1}\right) \geq a_{1}^{i} p_{1}-b_{1}^{i} q_{1}$. Since $p_{1} / q_{1} \geq \lambda_{1}^{i}$, this inequality is implied by the inequality $\lambda_{1}^{i} \geq\left(2 n-b_{1}^{i}\right) /\left(2 n-a_{1}^{i}\right)$ which is established by induction in exactly the way that (6.19) was established.

Finally, the case in which $\left(X_{1}^{*}, Y_{1}\right)$ lies in region VI must be considered. Examination of Table 2 shows that the only superscript possible is $j=n-2$, and so

$$
M\left(X_{1}^{*}, Y_{1}\right)=(n+1) p_{1}-q_{1}-\bar{x}_{1}^{i}+(n-1) \bar{u}_{1}^{i}-(n-1) y_{1}+w_{1} .
$$

The minimum of this expression is assumed at $Y_{1}=Y_{1}^{(1)}$. Since $M_{1}\left(X_{1}^{*}\right.$, $\left.Y_{1}^{(1)}\right)=V_{1}$, the proof of (4.2) is now concluded.

10. Verification, third part of sufficiency condition. The proof of (4.3) will clearly involve the computation of

$$
\begin{aligned}
\mathscr{C l}\left(X_{1}\right)=\alpha_{1} M_{1}\left(X_{1}, Y_{1}^{(1)}\right) & +\beta_{1} M_{1}\left(X_{1} Y_{1}^{(2)}\right) \\
& +\left(1-\alpha_{1}-\beta_{1}\right) M_{1}\left(X_{1}, Y_{1}^{(3)}\right) .
\end{aligned}
$$

Thus, for each $X_{1}$ it is necessary to know the case to which we are led by each of the points

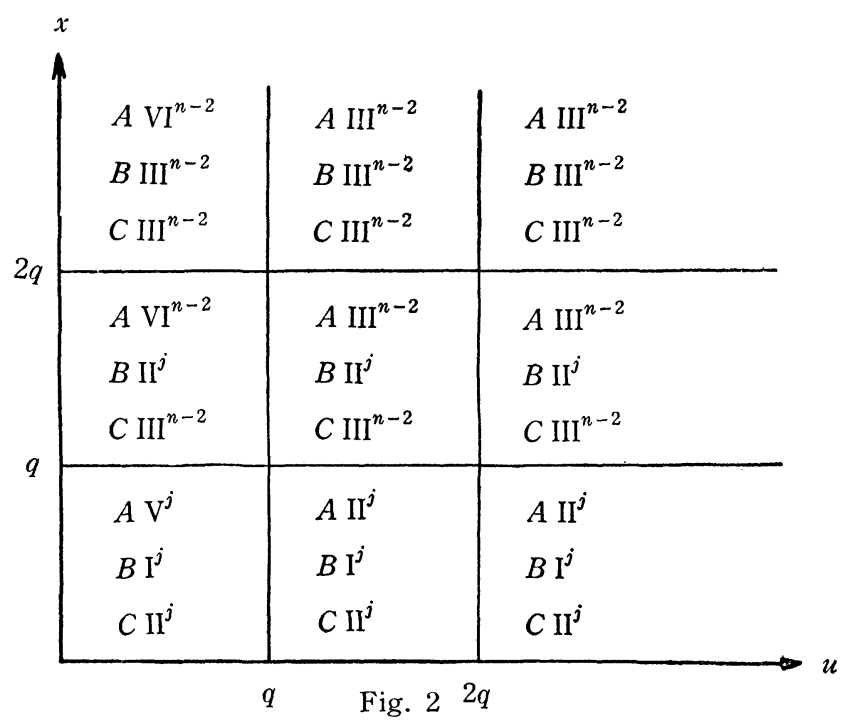




\section{$A: \quad\left(X_{1}, Y_{1}^{(1)}\right), \quad B:\left(X_{1}, Y_{1}^{(2)}\right), \quad C:\left(X_{1}, Y_{1}^{(3)}\right)$.}

Part of this information is tabulated in Fig. 2. In this tabulation, a symbol such as, say, $A V^{j}$ in a given region means that, for all $X_{1}$ in that region, $\left(X_{1}, Y_{1}^{(1)}\right)$ leads to case $V^{j}$. In some instances, the value of $j$ is indicated; in others, the determination of $j$ will be made in the discussions of Cases 1 and 2 below.

Case 1. $\lambda_{1}^{n-1} \leq p_{1} / q_{1}$.

First, the values assumed by the superscripts $j$ will be determined. In the case $\mathrm{BI}^{j}$, Table 2 shows that $p_{1} / q_{1}=p / q$; and since, by (6.16), $\lambda_{1}^{n-1} \geq \lambda^{n-2}$, it follows that $j=n-2$. Also, in the case $\mathrm{BII}^{j}$, we have $j=n-2$, because

$$
\frac{p}{q}=\frac{p_{1}}{2 q_{1}-x_{1}} \geq \frac{p_{1}}{q_{1}}, \quad q_{1} \leq x_{1} \leq 2 q_{1} .
$$

Similarly, in $A \mathrm{II}^{j}$ and $C \mathrm{II}^{j}$, we have $j=n-2$, because the relation $p / q=p_{1} /\left(q_{1}-x_{1}\right) \geq p_{1} / q_{1}$ holds there. In the case $A V^{j}$, the value of $j$ is determined by the ratio

$$
\lambda^{\jmath} \leq \frac{p}{q}=\frac{p_{1}-q_{1}+u_{1}}{q_{1}-x_{1}} \leq \lambda^{\jmath+1}, \quad j=1, \cdots, n-2 .
$$

The lines

$$
l^{3}: \lambda^{j} x_{1}+u_{1}=\lambda^{j} q_{1}-\left(p_{1}-q_{1}\right)
$$

form a finite pencil through the point $x_{1}=q_{1}, u_{1}=-\left(p_{1}-q_{1}\right)$. It follows from the monotonicity of the $\lambda$ 's that if a line $u_{1}=c$ with $c>-\left(p_{1}-q_{1}\right)$ is traversed from $x_{1}=q_{1}$ in the direction of decreasing $x_{1}$, then the lines of the pencil are encountered in order of decreasing $j$, with $l^{n-2}$ being the first line encountered. Thus, the lines $l^{j}$ divide the square $0 \leq x_{1} \leq q_{1}$, $0 \leq u_{1} \leq q_{1}$ into subregions over each of which a different superscript $j$

Table 3

Determination of Values of the coefficients $R$ and $S$

\begin{tabular}{c|c|c|c|c}
\hline \multicolumn{2}{c|}{ Region of $\left(x_{1}, u_{1}\right)$ plane } & $\begin{array}{c}\text { Region } \\
\text { Number }\end{array}$ & $R$ & $S$ \\
\hline$x_{1}$ & $u_{1}$ & $(1)$ & -1 & -1 \\
\hline $2 q_{1} \leq x_{1}$ & $u_{1} \geq q_{1}$ & 0 & -1 \\
\hline$q_{1} \leq x_{1} \leq 2 q_{1}$ & $u_{1} \geq q_{1}$ & $(2)$ & -1 \\
\hline $0 \leq x_{1} \leq q_{1}$ & $u_{1} \geq q_{1}$ & $(3)$ & $b^{n-2}-2$ & 0 \\
\hline $2 q_{1} \leq x_{1}$ & $u_{1} \leq q_{1}$ & $(4)$ & -1 & 0 \\
\hline$q_{1} \leq x_{1} \leq 2 q_{1}$ & $u_{1} \leq q_{1}$ & $(5)$ & 0 & $\frac{a^{j}}{n}-1$ \\
\hline $0 \leq x_{1} \leq q_{1}$ & $u_{1} \leq q_{1}$ & $(6)$ & $\frac{\left(b^{n-2}-1\right) b^{j}}{b^{n-2}}-1$ & \\
\hline
\end{tabular}


is applicable. The number of subregions depends on the ratio $p_{1} / q_{1}$. For sufficiently large values of this ratio, the entire square will have the superscript value $n-2$ associated with it. The important fact to be noted is that the region with superscript $n-2$ always exists and contains the line segment $x_{1}=q_{1}, 0 \leq u_{1} \leq q_{1}$.

Clearly, $\mathscr{C}\left(X_{1}\right)$ is continuous and is of the form

$$
\mathscr{C l}\left(X_{1}\right)=F\left(p_{1}, q_{1}\right)+R x_{1}+S u_{1},
$$

where $F\left(p, q_{1}\right)$ is a step function on the $\left(x_{1}, u_{1}\right)$ plane whose values are expressions involving the constants $a^{j}, b^{j}$ and the initial conditions $p_{1}, q_{1}$. Its exact form is of no concern here. The coefficients $R$ and $S$ are also step functions on the $\left(x_{1}, u_{1}\right)$ plane whose values involve the constants $a^{j}, b^{j}$. The information concerning $R$ and $S$ shown in Table 3 is easily obtained from Fig. 2, the preceding discussion, Table 2, and the definitions (7.2) of $\alpha_{1}$ and $\beta_{1}$.

The superscript $j$ in the entries for region (6) varies as the superscript in $A \mathrm{~V}^{j}$, and assumes the same values as the superscript in $A \mathrm{~V}^{j}$.

For each of the regions (1) through (6) of this table, the set of points at which the maximum of $\mathscr{C}\left(X_{1}\right)$ is achieved on that region is easily determined from the tabulated values of $R$ and $S$ in the region. It then follows from the continuity of $\mathscr{l l}\left(X_{1}\right)$ that the maximum of $\mathscr{C l}\left(X_{1}\right)$ is achieved at all points of the square $q_{1} \leq x_{1} \leq 2 q_{1}, 0 \leq u_{1} \leq q_{1}$ In particular, it is achieved at $\left(\bar{x}_{1}^{n-2}, \bar{u}_{1}^{n-2}\right)$, since by (7.3) this point is in the square. It now follows from (8.1) that

$$
\mathscr{l l}\left(X_{1}\right) \leq \mathscr{C}\left(X_{1}^{*}\right)=V_{1}\left(p_{1}, q_{1}\right) .
$$

Case 2. $\lambda_{1}^{i} \leq p_{1} / q_{1} \leq \lambda_{1}^{i+1}$,

$i=1,2, \cdots, n-2$

Under these initial conditions, Fig. 2 is modified from the outset as follows. Point $C$ is eliminated since we have $\alpha_{1}^{i}+\beta_{1}^{i}=1$ for $1 \leq i \leq n-2$; and the region $x_{1} \geq 2 q_{1}, u_{1} \geq q_{1}$ need not be considered since $p_{1} / q_{1}<3$. In determining the superscript $j$ and the modifications of Fig. 2, it will be convenient to distinguish two cases, namely $p_{1} / q_{1} \geq 2$ and $p_{1} / q_{1} \leq 2$.

Suppose that $p_{1} / q_{1} \geq 2$. The superscript $j$ in $B \mathrm{II}^{j}$ is determined by the ratio

$$
\frac{p}{q}=\frac{p_{1}}{2 q_{1}-x_{1}}
$$

where $q_{1} \leq x_{1} \leq \min \left[2 q_{1}, p_{1}-u_{1}\right]$. Thus $j$ is a nondecreasing step function of $x_{1}$ alone whose value at $x_{1}=2 q_{1}$ is $n-q$, and whose jumps occur at

$$
x_{1}=2 q_{1}-\frac{p_{1}}{\lambda^{j}},
$$


where $j$ is such that $2 q_{1}-p_{1} / \lambda^{j} \geq q_{1}$. At the jump points, $j$ is continuous from the right. Let $j_{0}$ denote the lowest value of the superscript $j$. This is clearly assumed at $x_{1}=q_{1}$, and the defining relation for $j_{0}$ can be taken as

$$
\lambda^{j_{0}} \leq \frac{p_{1}}{q_{1}} \lambda^{j_{0}+1}
$$

Since, by assumption and (6.18), $p_{1} / q_{1} \leq \lambda_{1}^{i+1}<\lambda^{i+1}$, it follows that $j_{0} \leq i$. It is also necessary to have some information concerning the superscript at $u_{1}=q_{1}, x_{1}=p_{1}-q_{1}$. Substitution of this value of $x_{1}$ into (10.2) gives the quantity $p_{1} /\left(3 q_{1}-p_{1}\right)$. It can be shown that $p_{1} /\left(3 q_{1}-p_{1}\right) \leq \lambda^{i+1}$, and hence it follows that $j \leq i$ at the point $x_{1}=p_{1}-q_{1}, u_{1}=q_{1}$.

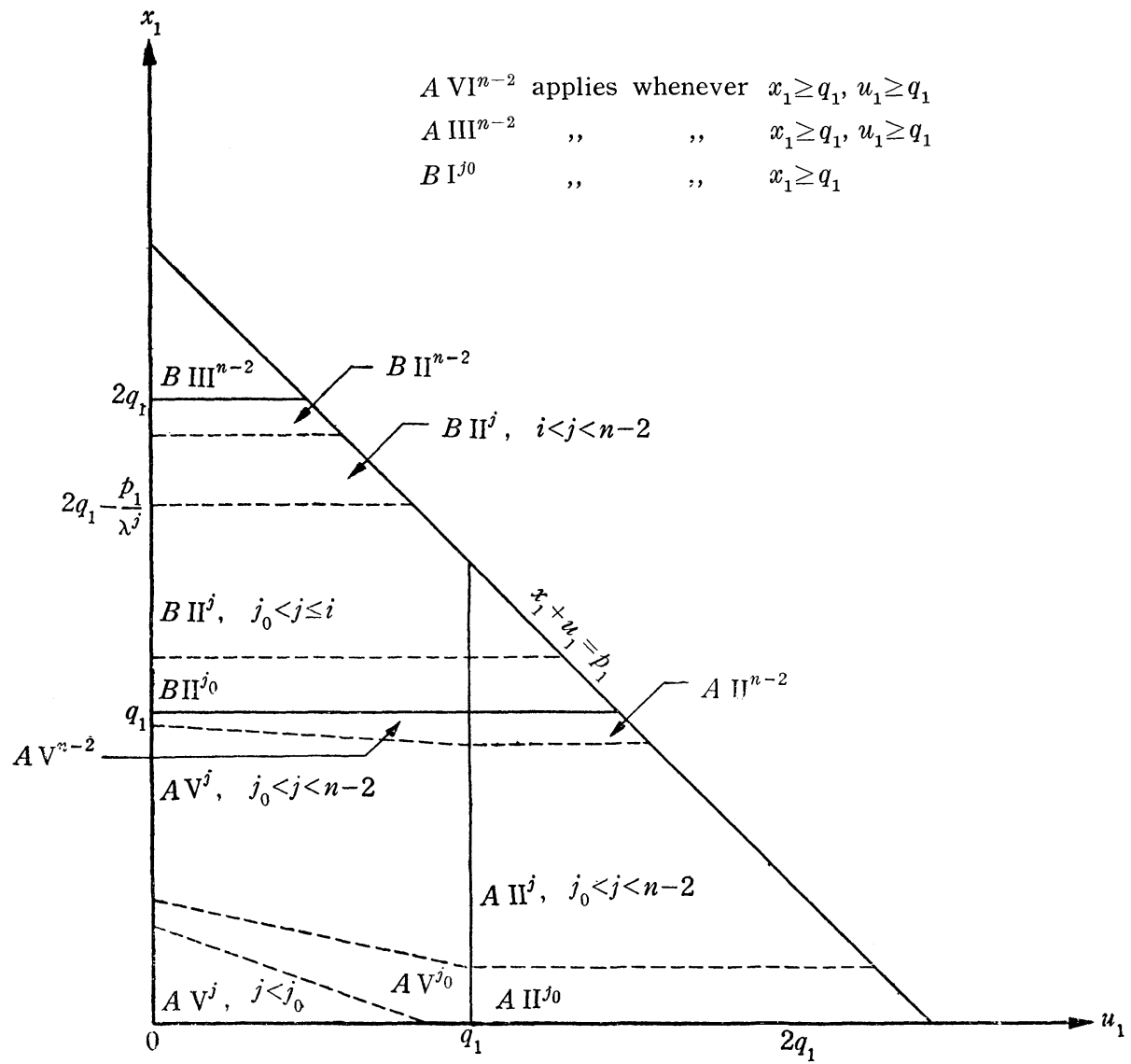

Fig. 3

The superscript $j$ in $B \mathrm{I}^{j}$ is determined by the ratio $p_{1} / q_{1}$; and, in view of (10.4), this makes $j=j_{0}$. In $A \mathrm{II}^{j}$ the superscript is determined by $p / q=p_{1} /\left(q_{1}-x_{1}\right)$. Thus $j$ is an increasing step function of $x_{1}$ alone, having value $j_{0}$ at $x_{1}=0$, and $n-2$ at $x_{1}=q_{1}$. The remarks made under Case 1 concerning $A \mathrm{~V}^{j}$ are applicable here, too. It is not difficult 
to see that the lines $l^{j}$ split up the square $0 \leq x_{1} \leq q_{1}, 0 \leq u_{1} \leq q_{1}$ as indicated in Fig. 3, which summarizes the foregoing discussion.

Suppose now that $p_{1} / q_{1} \leq 2$. Most of the remarks concerning the superscript $j$ in $B \mathrm{II}^{j}$ in the case $p_{1} / q_{1} \geq 2$ are also valid here. Now, however, $u_{1}$ cannot exceed $q_{1}$ when $x_{1} \geq q_{1}$, and so there is no need to discuss the point $u_{1}=q_{1}, x_{1}=p_{1}-q_{1}$. If $j_{1}$ denotes the maximum value of the superscript $j$, it no longer need be true that $j_{1}=n-2$. However, the relation $j_{1} \geqq i$ does hold. For, the maximum value of $p_{1} / q_{1}$ is $p_{1} /\left(2 q_{1}-p_{1}\right)$, and so the assertion $j_{1} \geq i$ is equivalent to $p_{1} /\left(2 q_{1}-p_{1}\right) \geq \lambda^{i}$. This relation, however, is easily established.

As before, the superscript in $B \mathrm{I}^{j}$ takes on the value $j_{0}$. In $A \mathrm{II}^{j}$, it is readily seen that the superscript $j$ is equal to $j_{0}$ at $x_{1}=0$ and increases to the maximum value of $j_{1}$. In $A \mathrm{~V}^{j}$, the remarks made in the discussion of $p_{1} / q_{1} \geq 2$ still hold, except that for $x_{1} \geq p_{1}-q_{1}$ the

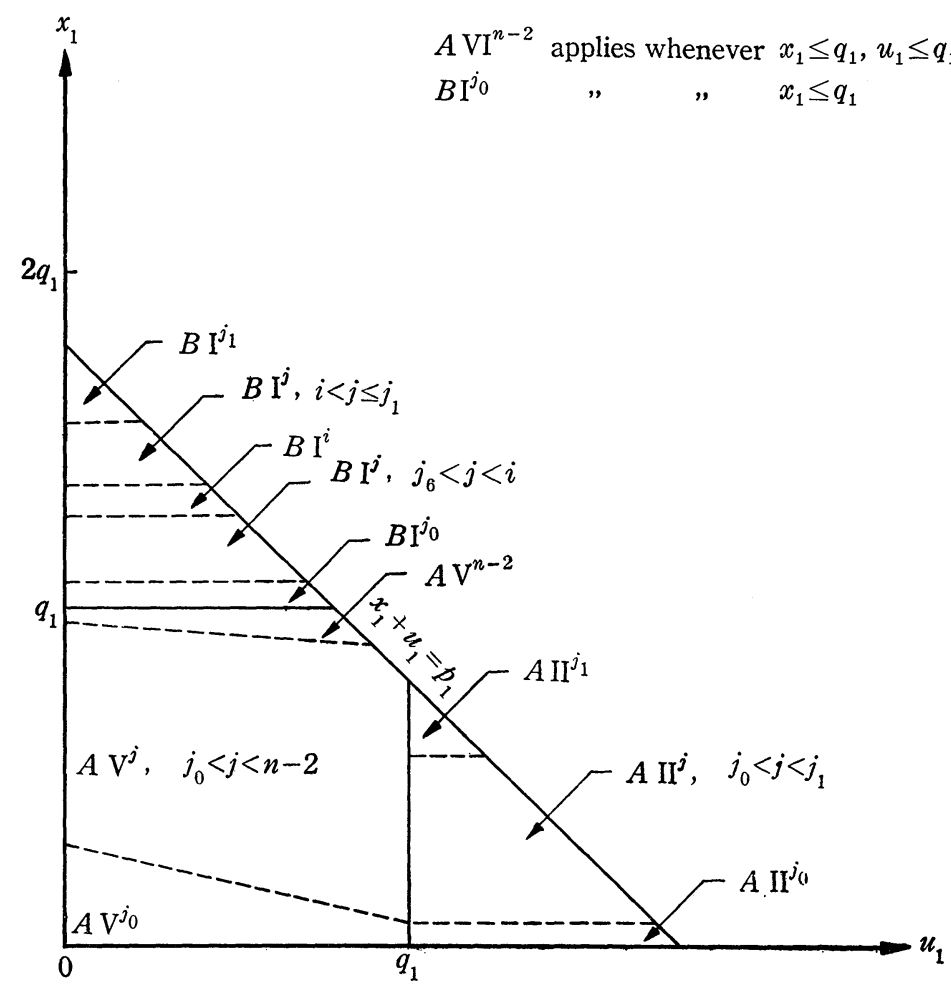

Fig. 4

regions are truncated by the line $x_{1}+u_{1}=p_{1}$. Furthermore, the smallest superscript involved in a truncated region is clearly $j_{1}$, and.so is greater than $i$. This information is summarized in Fig. 4.

Regardless of whether $p_{1} \geq 2 q_{1}$, or $p_{1} \leq 2 q_{1}$, we may write

$$
\mathscr{C l}\left(X_{1}\right)=H\left(p_{1}, q_{1}\right)+T x_{1}+U u_{1},
$$


where $H, T$, and $U$ are step functions having values that depend on the choice of $X_{1}$, but do not involve the variables $x_{1}$ or $u_{1}$. The functional values of $H, T$, and $U$ do involve the constants $a^{i}, b^{i}, a^{j}, b^{j}$, and those of $H$ involve $p_{1}$ and $q_{1}$ in addition. The superscripts $j$, of course, are determined by $X_{1}$. The values of $T$ and $U$ are shown in Table 4, and the regions of constancy are indicated. Clearly, the regions of constancy of $F$ coincide with those of $T$ and $U$.

Table 4

Determination of Values of the coefficients $T$ and $U$

\begin{tabular}{|c|c|c|c|c|}
\hline $\begin{array}{l}\text { Region of }(x, u) \\
\text { Plane }\end{array}$ & $\begin{array}{l}\text { Region } \\
\text { Number }\end{array}$ & $T$ & $U$ & Remarks \\
\hline $\begin{array}{l}2 q_{1} \leq x_{1} \\
u_{1} \leq q_{1}\end{array}$ & (1) & -1 & $\frac{n b^{i}}{b^{i}+n}-1$ & Only applies if $p_{1} \geq 2 q_{1}$ \\
\hline $\begin{array}{l}q_{1} \leq x_{1} \leq 2 q_{1} \\
q_{1} \leq u_{1}\end{array}$ & (2) & $\frac{n b^{j}}{b^{i}+n}-1$ & -1 & $\begin{array}{c}\text { Only applies if } p_{1} \geq 2 q_{1} \text {; exponent } \\
j \text { varies as superscript in } B \mathrm{II}^{j}\end{array}$ \\
\hline $\begin{aligned} q_{1} \leq x_{1} & \leq 2 q_{1} \\
u_{1} & \leq q_{1}\end{aligned}$ & (3) & $\frac{n b^{j}}{b^{i}+n}-1$ & $\frac{n b^{i}}{b^{i}+n}-1$ & $\begin{array}{l}\text { Exponent } j \text { varies as } \\
\text { superscript in } B \mathrm{II}^{j}\end{array}$ \\
\hline $\begin{aligned} 0 & \leq x_{1} \leq q_{1} \\
q_{1} & \leq u_{1}\end{aligned}$ & (4) & $\frac{b^{i} b^{j}}{b^{i}+n}-1$ & -1 & $\begin{array}{l}\text { Exponent } j \text { varies as } \\
\text { superscript in } A \mathrm{II}^{j}\end{array}$ \\
\hline $\begin{array}{r}0 \leq x_{1} \leq q_{1} \\
u_{1} \leq q_{1}\end{array}$ & (5) & $\frac{b^{i} b^{j}}{b^{i}+n}-1$ & $\frac{b^{i} a^{j}}{b^{i}+n}-1$ & $\begin{array}{l}\text { Exponent } j \text { varies as } \\
\text { superscript in } A \mathrm{~V}^{j}\end{array}$ \\
\hline
\end{tabular}

Suppose now that $p_{1} \geq 2 q_{1}$. Since $n b^{i}>n+b^{i}$, the maximum of $\mathscr{C l}\left(X_{1}\right)$ over region (1) of Table 4 occurs at $x_{1}=2 q_{1}, u_{1}=p_{1}-2 q_{1}$. Then the following four facts,

(a) $n b^{i}>b^{i}+n$,

(b) the $b^{j}$ 's are decreasing in $j$,

(c) the point $x_{1}=p_{1}, u_{1}=q_{1}$ lies in a set for which the superscript $j$ in $B \mathrm{II}^{j}$ does not exceed $i$, and

(d) the continuity of $\mathscr{C}\left(X_{1}\right)$, have the following implications: (a) the maximum of $\mathscr{C}\left(X_{1}\right)$ over region (2) and that part of region (3) lying below $x_{1}=p_{1}-q_{1}$ is attained at $x_{1}=p_{1}-q_{1}, u_{1}=q_{1}$; (b) the maximum of $\mathscr{C l}\left(X_{1}\right)$ over that part of region (2) lying above $x_{1}=p_{1}-q_{1}$ is attained at all points of the line $x_{1}+u_{1}=p_{1}$ that lie in the strip for which the superscript in $B \mathrm{II}^{j}$ assumes the value $i$. Denote this set of points by $\mathscr{E}$. Again appealing to the continuity of $\mathscr{C}\left(X_{1}\right)$, we see that the maximum of $\mathscr{C}\left(X_{1}\right)$ over all admissible $X_{1}$ for which $x_{1} \geq q_{1}$ is achieved on $\mathscr{E}$.

It is now asserted that $X_{1}^{*}$ lies in $\mathscr{E}$. In view of $(10.3)$, this is equivalent to showing that we have

$$
2 q_{1}-\frac{p_{1}}{\lambda^{i}} \leq \bar{x}_{1}^{i} \leq 2 q_{1}-\frac{p_{1}}{\lambda^{i+1}}, \quad i=1, \cdots, n-2 .
$$

The right-hand inequality follows immediately from (7.4) and the initial 
conditions. The left-hand inequality follows from the definition (7.1) of $\bar{x}_{1}^{i},(6.15)$, and the initial conditions.

Thus, it has been proved that

$$
\mathscr{H}\left(X_{1}^{*}\right) \leq \mathscr{L}\left(X_{1}\right)
$$

for all $X_{1}$ such that $x_{1} \geq q_{1}$. Since $\mathscr{L}\left(X_{1}^{*}\right)=V_{1}\left(p_{1}, q_{1}\right)$, to complete the proof of (4.3) in the case $\lambda_{1}^{i} \leq p_{1} / q_{1} \leq \lambda_{1}^{i+1}, p_{1} / q_{1} \geq 2$, it is sufficient to show that (10.6) holds for all $X_{1}$ such that $x_{1} \leq q_{1}$. From the form of $S$ in region (5) of Table 4 , it is clear that $\mathscr{K}\left(X_{1}\right)$ attains its maximum along the line $u_{1}=q_{1}$ whenever $x_{1} \leq q_{1}$. From the form of $R$ in this region, it is clear that if $b^{i} b^{n-2} \geq b^{i}+n$, then the maximum of $\mathscr{C}\left(X_{1}\right)$ is attained at $\left(x_{1}, u_{1}\right)=\left(q_{1}, q_{1}\right)$. Hence, $(10.6)$ follows for all $X_{1}$ in this event. On the other hand, if $b^{i} b^{n-2}<b^{i}+n$, then the maximum of $\mathscr{C}\left(X_{1}\right)$ will be attained at one of the points $x_{1}=q_{1}-p_{1} / \lambda^{j} \geq 0$, or $x_{1}=0$, of the line $u_{1}=q_{1}$. In this event, it can be shown by lengthy computation that (10.6) holds for such $X_{1}$. Thus (4.3) is established for $\lambda_{1}^{i} \leq p_{1} / q_{1} \leq \lambda_{1}^{i+1}, p_{1} \geq 2 q_{1}$. By similar methods, which will not be carried out here, (4.3) can be established for $p_{1} \leq 2 q_{1}$. Thus the validity of (4.3), and hence that of the theorem, is established.

\section{REFERENCES}

1. Leonard D. Berkovitz and Mevin Dresher, A game theory analysis of tactical air war, Operations Res. 7 (1959), 599-620.

2. J. von Neumann, Zur Theorie der Gesellschaftsspiele, Mat. Annalen, Vol. 100 (1928), 295-320.

3. J. Ville, Sur la théorie générale des jeux où intervient l'habilité des joueurs, Traite du Calcul des Probabilités et de ses Applications, ed. by E. Borel and Collaborators, 2 No. 5, Gauthier-Villars and Co., Paris, (1938), 105-113.

THE RAND CORPORATION 



\section{PACIFIC JOURNAL OF MATHEMATICS}

\section{EDITORS}

\author{
David Gilbarg \\ Stanford University \\ Stanford, California \\ F. H. Brownell \\ University of Washington \\ Seattle 5 , Washington
}

\author{
A. L. Whiteman \\ University of Southern California \\ Los Angeles 7, California \\ L. J. PAIGE \\ University of California \\ Los Angeles 24, California
}

\section{ASSOCIATE EDITORS}

\author{
E. F. BECKENBACH \\ T. M. CHERRY \\ D. DERRY
}
E. HEWITT
A. HORN
L. NACHBIN
M. OHTSUKA
H. L. ROYDEN
M. M. SCHIFFER

E. SPANIER

E. G. STRAUS

F. WOLF

\section{SUPPORTING INSTITUTIONS}

\author{
UNIVERSITY OF BRITISH COLUMBIA \\ CALIFORNIA INSTITUTE OF TECHNOLOGY \\ UNIVERSITY OF CALIFORNIA \\ MONTANA STATE UNIVERSITY \\ UNIVERSITY OF NEVADA \\ NEW MEXICO STATE UNIVERSITY \\ OREGON STATE COLLEGE \\ UNIVERSITY OF OREGON \\ OSAKA UNIVERSITY \\ UNIVERSITY OF SOUTHERN CALIFORNIA
}

\author{
STANFORD UNIVERSITY \\ UNIVERSITY OF TOKYO \\ UNIVERSITY OF UTAH \\ WASHINGTON STATE COLLEGE \\ UNIVERSITY OF WASHINGTON

AMERICAN MATHEMATICAL SOCIETY
CALIFORNIA RESEARCH CORPORATION
HUGHES AIRCRAFT COMPANY
SPACE TECHNOLOGY LABORATORIES
NAVAL ORDNANCE TEST STATION

Mathematical papers intended for publication in the Pacific Journal of Mathematics should be typewritten (double spaced), and the author should keep a complete copy. Manuscripts may be sent to any one of the four editors. All other communications to the editors should be addressed to the managing editor, L. J. Paige at the University of California, Los Angeles 24, California. ..

50 reprints per author of each article are furnished free of charge; additional copies may be obtained at cost in multiples of 50 .

The Pacific Journal of Mathematics is published quarterly, in March, June, September, and December. The price per volume (4 numbers) is $\$ 12.00$; single issues, $\$ 3.50$. Back numbers are available. Special price to individual faculty members of supporting institutions and to individual members of the American Mathematical Society: $\$ 4.00$ per volume; single issues, $\$ 1.25$.

Subscriptions, orders for back numbers, and changes of address should be sent to Pacific Journal of Mathematics, 2120 Oxford Street, Berkeley 4, California.

Printed at Kokusai Bunken Insatsusha (International Academic Printing Co., Ltd.), No. $6_{4}$ 2-chome, Fujimi-cho, Chiyoda-ku, Tokyo, Japan.

PUBLISHED BY PACIFIC JOURNAL OF MATHEMATICS, A NON-PROFIT CORPORATION

The Supporting Institutions listed above contribute to the cost of publication of this Journăl, but they are not owners or publishers and have no responsibility for its content or policies. 


\section{Pacific Journal of Mathematics}

\section{Vol. 10, No. $3 \quad$ November, 1960}

Glen Earl Baxter, An analytic problem whose solution follows from a simple

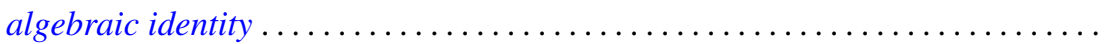

Leonard D. Berkovitz and Melvin Dresher, A multimove infinite game with linear payoff. .

Earl Robert Berkson, Sequel to a paper of A. E. Taylor ......................

Gerald Berman and Robert Jerome Silverman, Embedding of algebraic systems.... 767

Peter Crawley, Lattices whose congruences form a boolean algebra . . . . . ...... 777

Robert E. Edwards, Integral bases in inductive limit spaces . . . . . . . . . . . . . . .

Daniel T. Finkbeiner, II, Irreducible congruence relations on lattices . . . . . . . . . .

William James Firey, Isoperimetric ratios of Reuleaux polygons . . . . . . . . . . . 787

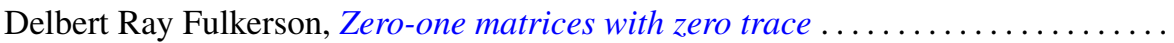

Leon W. Green, A sphere characterization related to Blaschke's conjecture........

Israel (Yitzchak) Nathan Herstein and Erwin Kleinfeld, Lie mappings in

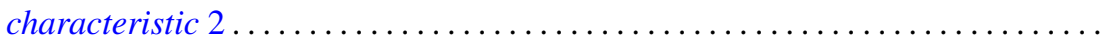

Charles Ray Hobby, A characteristic subgroup of a $p$-group .................

R. K. Juberg, On the Dirichlet problem for certain higher order parabolic

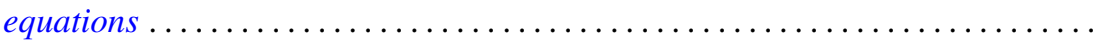

Melvin Katz, Infinitely repeatable games ......................

Emma Lehmer, On Jacobi functions . . . . . . . . . . . . . . . . . . . . . . . . .

D. H. Lehmer, Power character matrices

Henry B. Mann, A refinement of the fundamental theorem on the density of the sum of two sets of integers.

Marvin David Marcus and Roy Westwick, Linear maps on skew symmetric matrices: the invariance of elementary symmetric functions . .

Richard Dean Mayer and Richard Scott Pierce, Boolean algebras with ordered

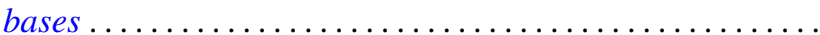

Trevor James McMinn, On the line segments of a convex surface in $E_{3} \ldots$

Frank Albert Raymond, The end point compactification of manifolds ..

Edgar Reich and S. E. Warschawski, On canonical conformal maps of regions of arbitrary connectivity

Marvin Rosenblum, The absolute continuity of Toeplitz's matrices...

Lee Albert Rubel, Maximal means and Tauberian theorems . .

Helmut Heinrich Schaefer, Some spectral properties of positive linear operators

Jeremiah Milton Stark, Minimum problems in the theory of pseudo-conformal transformations and their application to estimation of the curvature of the invariant metric.

Robert Steinberg, The simplicity of certain groups ...

Hisahiro Tamano, On paracompactness. .

Angus E. Taylor, Mittag-Leffler expansions and spectral theory .

Marion Franklin Tinsley, Permanents of cyclic matrices ...... . 